\title{
The Impact of COVID-19 Pandemic on Admission Times, Manual Detorsion Rates and Outcomes in Testicular Torsion
}

\author{
Emre Tokuç ${ }^{1}$, Ilker Artuk ${ }^{1}$, Ridvan Kayar $^{1}$, Emrah Özsoy ${ }^{2}$, Ramazan Topaktas ${ }^{1}$, Yavuz \\ Bastug $^{1}$, and Mehmet Akyüz ${ }^{1}$ \\ ${ }^{1}$ University of Health Sciences, Haydarpasa Numune SUAM

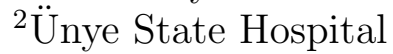

September 3, 2021

\begin{abstract}
Aim: This study aims to assess the admission times, manual detorsion rates and outcomes of the testicular torsion cases during COVID-19 pandemic. Methods: A retrospective analysis of the testicular torsion cases was done through our hospital's electronic archive. Patients with were distributed into two groups as "COVID-19 era" (between 11th of March 2020 - March 2021) and "pre-COVID-19 era" (between January 2018 and 10th of March 2020). Time between the onset of symptoms and the moment of detorsion was calculated as the duration of ischemia. Two groups were compared in terms of ischemia duration, manual detorsion rates and operational outcomes. Results: No statistically significant differences was detected between groups in terms of ischemia duration, manual detorsion rates and surgical procedure type (all p $>0.05$ ). During the COVID-19 pandemic, the number and percentage of manual detorsions have increased, resulting in a numerical decrease in orchiectomies. Conclusions: Conditions caused by COVID-19 may prompt urologists to manual detorsion more to set up safer surgical environment. Moreover, this approach may result in a decrease in orchiectomy rates by saving time and preserving the vascularity of the testis. Further studies with larger samples sizes and meta-analyses may support this concept. Keywords: testicular torsion, COVID-19, manual detorsion, orchiectomy, emergency
\end{abstract}

The Impact of COVID-19 Pandemic on Admission Times, Manual Detorsion Rates and Outcomes in Testicular Torsion

\section{ABSTRACT}

Aim: This study aims to assess the admission times, manual detorsion rates and outcomes of the testicular torsion cases during COVID-19 pandemic.

Methods: A retrospective analysis of the testicular torsion cases was done through our hospital's electronic archive. Patients with were distributed into two groups as "COVID-19 era" (between $11^{\text {th }}$ of March 2020 March 2021) and "pre-COVID-19 era" (between January 2018 and 10 ${ }^{\text {th }}$ of March 2020). Time between the onset of symptoms and the moment of detorsion was calculated as the duration of ischemia. Two groups were compared in terms of ischemia duration, manual detorsion rates and operational outcomes.

Results: No statistically significant differences was detected between groups in terms of ischemia duration, manual detorsion rates and surgical procedure type (all $\mathrm{p}>0.05$ ). During the COVID-19 pandemic, the number and percentage of manual detorsions have increased, resulting in a numerical decrease in orchiectomies.

Conclusions: Conditions caused by COVID-19 may prompt urologists to manual detorsion more to set up safer surgical environment. Moreover, this approach may result in a decrease in orchiectomy rates by saving time and preserving the vascularity of the testis. Further studies with larger samples sizes and meta-analyses may support this concept. 
Keywords: testicular torsion, COVID-19, manual detorsion, orchiectomy, emergency

\section{What's Already Known About This Topic?}

Testicular torsion is an organ-threatening pathology in urology practice in which time has a critical role. Delay in surgical treatment as detorsion of the torted testicle will lead to an organ loss. Conditions brought with the COVID-19 pandemic might complicate the admission times, leading to an undesirable increase in orchiectomies.

\section{What Does This Article Add?}

Although the admission times and the outcomes of testicular torsion cases did not vary during the pandemic, there was an increasing trend to manual detorsion for overcoming the emergency temporarily. This approach has led to a numerical decrease in orchiectomies compared with pre-pandemic era.

\section{Introduction}

COVID-19 pandemic which outbroke in December 2019 has caused disruption of both emergency and routine urological healthcare services around the world ${ }^{1,2}$. Through this period of worldwide health crisis, urologists have adapted by trying to take protective measures while striving not to interrupt their practice ${ }^{3}$. In our country, first COVID-19 case has been reported on $11^{\text {th }}$ of March, 2020 and since then; the daily workload of urologists has dramatically dropped. Although this decrease, nondeferrable emergent interventions were prioritized and continued ${ }^{4}$.

Testicular torsion is an acute urgent scrotal pathology in which time is of great importance in preserving the testis. Testis, being an end-organ, is vulnerable to ischemia and the time of ischemia is directly proportional with the risk of testicular atrophy and dysfunction ${ }^{5}$. Early admission is a critical factor to prevent these negative outcomes which leads to organ loss. Management of testicular torsion is a race against time even if in normal conditions. In this extraordinary COVID-19 pandemic which affects every aspect of clinical practice, approach to cases with testicular torsion becomes even more crucial.

The aim of our study is to evaluate the admission times, our approach and orchiectomy rates of patients diagnosed with testicular torsion during COVID-19 pandemic in our tertiary referral educational urology clinic.

\section{Materials \& Methods}

This study has been approved by the Clinical Studies Ethics Board of University of Health Sciences, Haydarpasa Numune SUAM (Decision Number: HNEAH-KAEK 2021/KK/138) and the study was conducted in compliance with the Declaration of Helsinki. Testicular torsion cases were retrospectively analyzed through our hospital's electronic archive system and patients were distributed into 2 groups as "COVID-19 era" (between 11 ${ }^{\text {th }}$ of March 2020 - March 2021) and "pre-COVID-19 era" (between January 2018 and $10^{\text {th }}$ of March 2020). Ages and laterality of the patients were recorded. Differential diagnosis of all patients was done with scrotal Doppler ultrasound. A written informed consent was taken from all patients prior to procedures. Patients whom manual detorsion were done were recorded and the vascularity of testis was confirmed with scrotal Doppler ultrasound after the procedure. The duration of ischemia was calculated in hours by considering the time between the onset of symptoms and the moment of detorsion (manual or operational). All perioperative findings and applied procedures were recorded. All orchiectomy materials were sent to the pathology department and the results were confirmed as necrotic tissues.

Since preoperative COVID-19 testing could not be done in patients who were operated immediately after diagnosis, emergency operations during pandemic were managed as the operations of COVID-19 positive patients, with taking preventive measures such as N95 masks and necessary personal protective equipments. Preoperative PCR (polymerase-chain reaction) testing was requested to patients whose manual detorsion were successful. Patients who tested negative for COVID-19 were electively operated under safer conditions.

Statistical Analysis 
Statistical Package for the Social Science (SPSS Inc, Chicago, IL, USA) version 22.0 was used for the statistical analysis. Differences between independent groups containing non-parametric variables which are not normally distributed within were compared with Mann-Whitney $\mathrm{U}$ test and $\mathrm{p}<0.05$ was considered as statistically significant.

\section{Results}

There were 31 testicular torsion cases, 13 while COVID-19 pandemic and 18 in pre-COVID era. The average age of all patients was $19.45 \pm 6.47$. The average age was 18.62 \pm 4.48 in COVID-19 group and 20.06 \pm 7.66 in pre-COVID-19 group. There was no significant difference between the groups in terms of age $(\mathrm{p}=0.441)$.

Generally, the average duration of ischemia was $19.03 \pm 22.83$ hours. The average time of ischemia was $19.46 \pm 31.54$ hours during the pandemic and 18.72 \pm 14.75 hours before the COVID-19 outbreak. No significant difference was detected between the groups in terms of ischemia time $(\mathrm{p}=0.115)$.

There was only one case $(5.6 \%)$ which manual detorsion was done in the pre-COVID-19 group. During the pandemic, four $(30.8 \%)$ patients underwent manual detorsion prior to elective surgery. There was no significant difference between groups in terms of patients underwent manual detorsion $(\mathrm{p}=0.242)$.

When the operative findings and outcomes are taken into consideration;

In the pre-COVID-19 era, out of 18 patients, six (33.3\%) underwent surgical detorsion + bilateral testicular orchiopexy because the testis was viable. In seven patients (38.9\%), necrotic testicular tissue was observed and orchiectomy + contralateral testicular orchiopexy was performed. In five patients, only bilateral orchiopexy was done. Four of these five patients had incomplete/partial torsion in the scrotal Doppler ultrasound prior to surgery. One patient underwent manual detorsion which was confirmed with ultrasound, so bilateral orchiopexy was done electively.

During the COVID-19 pandemic, four out of 13 patients (30.8\%) underwent surgical detorsion + bilateral testicular orchiopexy, three $(23.1 \%)$ underwent orchiectomy + contralateral testicular orchiopexy and six $(46.2 \%)$ underwent only bilateral orchiopexy. In two patients who underwent bilateral orchiopexy, incomplete/partial torsion was detected as preoperative ultrasound finding. In four patients, emergent manual detorsion was performed and after the negative COVID-PCR test results, bilateral orchiopexy was done electively.

Concerning the operative outcomes of these patients, there was no significant difference between two groups in terms of surgical procedure $(\mathrm{p}=0.514)$. (Table 1$)$

\section{Discussion}

Concerns about the contagiousness and mortalities of COVID-19 has led most governments take measures like lockdowns, stay-at-home and social distancing suggestions ${ }^{6}$. In hospitals, daily routine healthcare services were heavily interrupted, either the hospitals were completely converted into quarantine clinics or admitted reduced number of patients. There are many studies indicating significant delay in patients with urgent conditions in emergency departments such as cardiovascular emergencies ${ }^{7,8}$. Also, studies conducted in pediatric emergency clinics assert the fact that number of patients has significantly decreased during COVID19 pandemic and the main reason for this is the fear of infection and the protectionist instinct of the families 9,10 . In this study, the aggravated, acute and severe presentation of testicular torsion may be the reason not having any significant differences between ages and duration of symptoms between the groups. Similar to this study, Littman et al. and Nelson et al. found no delay in the time of presentation of testicular torsion cases during COVID-19 pandemic ${ }^{11,12}$. On the other hand, a very recent multicenter study from the Western Pediatric Urology Consortium (WPUC) suggests significantly longer presentation times of testicular torsion cases during the pandemic ${ }^{13}$.

In testicular torsion, ischemia duration and torsion degree are the most important factors in preserving the functional features of the testis ${ }^{14}$. Irreversible damage starts after four to six hours and affects both the spermatogenesis and the endocrine functions ${ }^{15,16}$. Also, it has been suggested that unilateral testicular 
torsion may harm the spermatogenesis in the contralateral testis through ischemia-reperfusion metabolites as an early mechanism and by autoimmune response in latter stages ${ }^{17}$. A study comparing the time to treat and duration of symptoms in respect of testicular salvage indicates a clear significance in conserving the testis in the group with duration of symptoms below 24 hours while there was no significance in time to treat ${ }^{18}$. Concerning all the issues above, immediate intervention is recommended. In regards to prompt management, manual detorsion has been described by Nash in 1893, has been proposed as urgent procedure reducing the duration of ischemia of torsioned testis ${ }^{19}$. Various studies in literature suggests that this method may be an alternative to exploration and surgical detorsion ${ }^{20-22}$. On the other hand, some studies in literature suggest the risk of residual torsion and subsequent testicular atrophy ${ }^{23,24}$. In this study, although there are no significant differences between groups, the number of manual detorsions has increased throughout the COVID-19 era. This result may be interpreted as the self-protective instinct of the surgeon providing an urgent and temporary management to a testicular torsion case which is COVID-19 suspicious. All patients with prior manual detorsion in this study have been confirmed with scrotal Doppler ultrasound and also operated under elective and COVID-PCR negative conditions for bilateral orchiopexy.

There were no significant differences between the groups in terms of surgical approach and orchiectomy rates. Since there were no significant differences in the admission times of the patients, this result could be predicted. Both Littman et al. and Nelson et al. showed no significant alterations in orchiectomy rates of testicular torsion cases during the pandemic which this study was consistent with ${ }^{11,12}$. Contrarily, in the multicenter study by WPUC, significantly delayed patients during the pandemic resulted in a non-significant but numerically higher percentages of orchiectomies ${ }^{13}$. In this study, during the COVID-19 pandemic, the number and percentage of orchiectomies have decreased numerically but not significantly. The increase in the number of manual detorsions may have contributed to this result. In addition to providing a remedy to the patient's pain and saving time for the surgeon, the manual detorsion procedure have been shown to have an organ-preserving aspect. This approach may also be implemented on daily practice to reduce orchiectomies.

Even though contralateral orchiopexy of the testis is controversial, there is still evidence in the literature indicating the risk of future torsions ${ }^{25}$. Hence, this approach is recommended to avoid the possible risk. All patients included in our study underwent contralateral orchiopexy in direction of the current guidelines and recommendations.

Emergency surgeries during COVID-19 pandemic are a great risk for the surgeon and the whole staff in the operating room. Both in urology and other aspects of surgery, biosecurity measures, recommendations and guidelines are continuously being reported about this subject ${ }^{3,26,27}$. Also a report by Parikh et al. shows in order not to compromise the outcome of testicular torsion, COVID-PCR testing results cannot be waited and all protective measures should be taken as if the patient is COVID-19 positive ${ }^{28}$.

Although this stands out to be the only study evaluating manual detorsion rates in testicular torsion during the COVID-19 era, the main limitation is its retrospective nature. Also, due to the short time intervals, especially in COVID-19 era which is 9 months in this study, number of patients is limited to reach definitive, descriptive and significant results. Randomized, prospective studies with increased number of participants may end up in statistical significance.

Even though there were no significant differences between pre-COVID-19 era and during the COVID-19 crisis in terms of admission times, the approach to testicular torsion and surgical outcomes, conditions caused by COVID-19 may prompt urologists to manual detorsion more to set up safer surgical environment. Additionally, this approach may result in a decrease in orchiectomy rates by saving time and preserving the vascularity of the testis.

\section{References}

1. Chung HS, Lee DE, Kim JK, Yeo IH, Kim C, Park J, et al. Revised Triage and Surveillance Protocols for Temporary Emergency Department Closures in Tertiary Hospitals as a Response to COVID-19 Crisis in Daegu Metropolitan City. J Korean Med Sci 2020;35:e189. 
2. Rocco B, Sighinolfi MC, Sandri M, Altieri V, Amenta M, Annino F, et al. The dramatic COVID 19 outbreak in Italy is responsible of a huge drop of urological surgical activity: a multicenter observational study. BJU Int 2021;127:56-63.

3. Amparore D, Campi R, Checcucci E, Sessa F, Pecoraro A, Minervini A, et al. Forecasting the Future of Urology Practice: A Comprehensive Review of the Recommendations by International and European Associations on Priority Procedures During the COVID-19 Pandemic. Eur Urol Focus 2020;6:1032-48.

4. Bozkurt O, Sen V, Irer B, Sagnak L, Onal B, Tanidir Y, et al. Nation-wide analysis of the impact of Covid-19 pandemic on daily urology practice in Turkey. Int J Clin Pract 2021;75:e13735.

5. Jacobsen FM, Rudlang TM, Fode M, Ostergren PB, Sonksen J, Ohl DA, et al. The Impact of Testicular Torsion on Testicular Function. World J Mens Health 2020;38:298-307.

6. Hale T, Petherick A, Phillips T, Webster S. Variation in government responses to COVID-19. Blavatnik school of government working paper 2020;31:2020-11.

7. Pines JM, Zocchi MS, Black BS, Celedon P, Carlson JN, Moghtaderi A, et al. The effect of the COVID-19 pandemic on emergency department visits for serious cardiovascular conditions. The American journal of emergency medicine 2021;47:42-51.

8. Range G, Hakim R, Beygui F, Angoulvant D, Marcollet P, Godin M, et al. Incidence, delays, and outcomes of STEMI during COVID-19 outbreak: Analysis from the France PCI registry. J Am Coll Emerg Physicians Open 2020.

9. Walker DM, Tolentino VR. COVID-19: the effects on the practice of pediatric emergency medicine. Pediatr Emerg Med Pract 2020;17:1-15.

10. Dopfer C, Wetzke M, Zychlinsky Scharff A, Mueller F, Dressler F, Baumann U, et al. COVID-19 related reduction in pediatric emergency healthcare utilization - a concerning trend. BMC Pediatr 2020;20:427.

11. Littman AR, Janssen KM, Tong L, Wu H, Wang MD, Blum E, et al. Did COVID-19 Affect Time to Presentation in the Setting of Pediatric Testicular Torsion? Pediatr Emerg Care 2021;37:123-5.

12. Nelson CP, Kurtz MP, Logvinenko T, Venna A, McNamara ER. Timing and outcomes of testicular torsion during the COVID-19 crisis. Journal of pediatric urology 2020;16:841 e1- e5.

13. Holzman SA, Ahn JJ, Baker Z, Chuang KW, Copp HL, Davidson J, et al. A multicenter study of acute testicular torsion in the time of COVID-19. Journal of pediatric urology 2021.

14. Feng S, Yang H, Lou Y, Ru W, Wang A, Liu W. Clinical Characteristics of Testicular Torsion and Identification of Predictors of Testicular Salvage in Children: A Retrospective Study in a Single Institution. Urologia internationalis 2020;104:878-83.

15. Arap MA, Vicentini FC, Cocuzza M, Hallak J, Athayde K, Lucon AM, et al. Late hormonal levels, semen parameters, and presence of antisperm antibodies in patients treated for testicular torsion. J Androl 2007;28:528-32.

16. Romeo C, Impellizzeri P, Arrigo T, Antonuccio P, Valenzise M, Mirabelli S, et al. Late hormonal function after testicular torsion. Journal of pediatric surgery 2010;45:411-3.

17. Xia Z, Hu J, Han L, Xia Q, Shao F, Lin X. Effect of unilateral testicular torsion on contralateral testis in a rat model. Pediatr Surg Int 2020;36:529-36.

18. Morin OA, Carr MG, Holcombe JM, Bhattacharya SD. Optimal Predictor of Gonadal Viability in Testicular Torsion: Time to Treat Versus Duration of Symptoms. J Surg Res 2019;244:574-8.

19. Nash WG. Acute torsion of spermatic cord: reduction: immediate relief. The British Medical Journal 1893:742-3. 
20. Demirbas A, Demir DO, Ersoy E, Kabar M, Ozcan S, Karagoz MA, et al. Should manual detorsion be a routine part of treatment in testicular torsion? BMC Urol 2017;17:84.

21. Dias Filho AC, Oliveira Rodrigues R, Riccetto CL, Oliveira PG. Improving Organ Salvage in Testicular Torsion: Comparative Study of Patients Undergoing vs Not Undergoing Preoperative Manual Detorsion. The Journal of urology 2017;197:811-7.

22. Siu Uribe A, Garrido Perez JI, Vazquez Rueda F, Ibarra Rodrigue MR, Murcia Pascual FJ, Ramnarine Sanchez SD, et al. [Manual detorsion and elective orchiopexy as an alternative treatment for acute testicular torsion in children]. Cir Pediatr 2019;32:17-21.

23. Sessions AE, Rabinowitz R, Hulbert WC, Goldstein MM, Mevorach RA. Testicular torsion: direction, degree, duration and disinformation. The Journal of urology 2003;169:663-5.

24. Gunes M, Umul M, Altok M. Atypical Testicular Torsion: We Must be Cautious in the Course of Manual Detorsion. Pediatr Emerg Care 2016;32:691-2.

25. Moore SL, Chebbout R, Cumberbatch M, Bondad J, Forster L, Hendry J, et al. Orchidopexy for Testicular Torsion: A Systematic Review of Surgical Technique. Eur Urol Focus 2020.

26. Coimbra R, Edwards S, Kurihara H, Bass GA, Balogh ZJ, Tilsed J, et al. European Society of Trauma and Emergency Surgery (ESTES) recommendations for trauma and emergency surgery preparation during times of COVID-19 infection. Eur J Trauma Emerg Surg 2020;46:505-10.

27. de la Reza MT, Autran-Gomez AM, Tardio GU, Bolanos JA, Rivero JCG. Emergency Surgery in Urology during the COVID-19 Pandemic. International braz j urol : official journal of the Brazilian Society of Urology 2020;46:201-6.

28. Parikh M, Fiadjoe J, Black S. Emergency surgery during the COVID-19 pandemic. Paediatr Anaesth 2020;30:728-9.

Tables

Table 1 : Characteristics and results of testicular torsion cases

\begin{tabular}{lllll}
\hline & $\begin{array}{l}\text { pre-COVID-19 } \\
\text { era }\end{array}$ & COVID-19 era & Total & p \\
\hline $\begin{array}{l}\text { Number of } \\
\text { Patients }\end{array}$ & 18 & 13 & 31 & \\
$\begin{array}{l}\text { Age (years) } \\
\text { Laterality } \\
\text { (Right / Left) }\end{array}$ & $20.06 \pm 7,66$ & $18.62 \pm 4.48$ & $19.45 \pm 6.47$ & 0.441 \\
$\begin{array}{l}\text { Duration of } \\
\text { Ischemia } \\
\text { (hours) }\end{array}$ & $18.72 \pm 14.75$ & $4 / 9$ & $17 / 14$ & \\
$\begin{array}{l}\text { Manual } \\
\begin{array}{l}\text { Detorsion (n) } \\
\text { (\%) }\end{array}\end{array}$ & $19.46 \pm 31.54$ & $19.03 \pm 22.83$ & 0.115 \\
$\begin{array}{l}\text { Orchiectomy }+ \\
\text { Contralateral }\end{array}$ & $7(38.9 \%)$ & $4(30.8 \%)$ & $5(16.1 \%)$ & \\
$\begin{array}{l}\text { Orchiopexy (n) } \\
\text { (\%) }\end{array}$ & & $3(23.1 \%)$ & $10(32.3 \%)$ & \\
$\begin{array}{l}\text { Detorsion }+ \\
\text { Bilateral } \\
\text { Orchiopexy (n) } \\
\text { (\%) }\end{array}$ & $6(33.3 \%)$ & & & \\
\end{tabular}




\begin{tabular}{lllll}
\hline & $\begin{array}{l}\text { pre-COVID-19 } \\
\text { era }\end{array}$ & COVID-19 era & Total & p \\
\hline $\begin{array}{l}\text { Bilateral } \\
\begin{array}{l}\text { Orchiopexy (n) } \\
(\%)\end{array}\end{array}$ & $5(27.8 \%)$ & $6(46.2 \%)$ & $11(35.5 \%)$ & \\
\hline
\end{tabular}

\section{Hosted file}

Table 1.docx available at https://authorea.com/users/313370/articles/536199-the-impact-ofcovid-19-pandemic-on-admission-times-manual-detorsion-rates-and-outcomes-in-testiculartorsion 